\title{
PREVALENCE OF HELICOBACTER PYLORI INFECTION IN PATIENTS WITH ACUTE PEPTIC ULCER BLEEDING IN A TERTIARY CARE HOSPITAL IN MANIPUR
}

\author{
Nongthombam Suraj Kumar1, Takhellambam Rebika Devi², Konjengbam Ghanachandra Singh ${ }^{3}$ \\ ${ }_{1}^{1}$ Assistant Professor (Gastroenterology), Department of Medicine, JNIMS, Imphal, Manipur, India. \\ ${ }^{2}$ Postgraduate Trainee, Department of Medicine, JNIMS, Imphal, Manipur, India. \\ 3 Professor and HOD, Department of Medicine, JNIMS, Imphal, Manipur, India.
}

\section{BACKGROUND}

ABSTRACT

Helicobacter pylori (H. pylori) is one of the commonest causes of peptic ulcer disease. Diagnosis and successful H. pylori eradication is important to prevent complications like recurrent peptic ulcer bleeding (PUB).

Aims and Objectives- This study aims to evaluate the prevalence of $\mathrm{H}$. pylori infection in patients with acute PUB.

\section{MATERIALS AND METHODS}

This study was carried out from September 2015 till August 2018 in patients with PUB admitted at JNIMS hospital who underwent endoscopic procedures in a prospective manner. RUTs were performed by taking mid-antral biopsy specimen.

Statistical Analysis- Baseline characteristics were presented as mean \pm standard deviation (SD) for continuous variables and as a frequency (percentage) for all variables. Chi square and two tailed Fischer's exact test were used where applicable.

\section{RESULTS}

Four hundred and sixty patients were included, of which duodenal ulcer bleeding comprised of 85\% (391), gastric ulcer $10.44 \%$ (48) and combined gastric and duodenal ulcer in 4.56\% (21). RUT was positive in 419 patients (91.08\%). Of the 48 gastric ulcer patients $82.6 \%$ (38) had positive RUT and of the 412 duodenal ulcer patients $92.47 \%$ (381) had positive RUT. H. pylori infection rates were high in patients with acute peptic ulcer bleeding patients.

\section{CONCLUSION}

This exploratory study showed the importance of actively looking for H. pylori infection in patients with acute peptic ulcer bleeds who are likely to rebleed if not treated with $\mathrm{H}$. pylori eradication regimes.

\section{KEY WORDS}

Peptic Ulcer Bleed, Helicobacter Pylori.

HOW TO CITE THIS ARTICLE: Kumar NS, Devi TR, Singh KG. Prevalence of helicobacter pylori infection in patients with acute peptic ulcer bleeding in a tertiary care hospital in Manipur. J. Evolution Med. Dent. Sci. 2018;7(50):5340-5342, DOI: $10.14260 /$ jemds/2018/1182

\section{BACKGROUND}

Helicobacter pylori ( $\mathrm{H}$. pylori) is one of the commonest causes of peptic ulcer disease, which may lead to severe complications such as peptic ulcer bleeding (PUB) or perforation. Diagnosis and successful H. pylori eradication is important to prevent recurrent PUB. ${ }^{1,2}$

The Maastricht IV consensus recommended that H. pylori eradication regime should be started at reintroduction of oral feeding in cases of bleeding ulcer as delaying treatment after discharge leads to reduced compliance or loss to follow-up without receiving treatment which can lead to recurrence of PUB. ${ }^{3}$

Of the various test to diagnose H. pylori infection, Rapid urease test (RUT) is a rapid, inexpensive and widely available method. Commercially available RUTs have a sensitivity and specificity above $90 \%$ and is one of the most commonly used modalities to diagnose $\mathrm{H}$. pylori infection in patients undergoing endoscopy. 4

'Financial or Other Competing Interest': None.

Submission 16-11-2018, Peer Review 28-11-2018,

Acceptance 30-11-2018, Published 10-12-2018.

Corresponding Author:

Nongthombam Suraj Kumar,

Department of Medicine,

JNIMS, Imphal-795005, Manipur, India.

E-mail: surajn30@yahoo.co.uk

DOI: $10.14260 /$ jemds/2018/1182

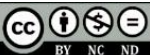

The prevalence of $\mathrm{H}$. pylori infection in Indian population is estimated to be about $80 \%$ but the data in patients with peptic ulcer bleeding is meagre. 5

Manipur is one of the North Eastern states of India sharing international boundary with Myanmar which has a very unique topography, culture and dietary habits and there are no existing data on the prevalence of H. pylori infection in this region. And also, there have been no studies on the prevalence of $\mathrm{H}$. pylori infection in Manipuri patients with acute PUB. This exploratory study was undertaken to evaluate the prevalence of $\mathrm{H}$. pylori infection in patients with acute PUB.

\section{MATERIAL AND METHODS Patient Population}

Consecutive patients with acute upper Gastrointestinal bleeding and endoscopically confirmed gastric and duodenal ulcers admitted at JNIMS Medicine ward between September 2015 till August 2018 were included in this study. Patients previously treated for $\mathrm{H}$. pylori infection or who gave a clear history of using proton pump inhibitors with two weeks or antibiotics within the past four weeks of endoscopy were excluded. Permission from Ethics committee JNIMS was obtained and written consent was obtained from all study participants. 


\section{Study Design}

This was a hospital based cross sectional study. Consecutive patients with acute PUB admitted at JNIMS Medicine ward were recruited

\section{Study location}

This was a tertiary care teaching hospital-based study done in Department of General Medicine at Jawaharlal Nehru Institute of Medical Sciences, Imphal, Manipur.

\section{Data Collection}

Patients admitted at JNIMS Medicine ward with the presentation of hematemesis or melena were resuscitated and prepared for upper gastrointestinal endoscopy. During endoscopy, the presence of blood in the upper gastrointestinal tract and the recent stigmata of bleeding ulcers were recorded. Endoscopic intervention was performed if high-risk lesions (i.e., spurting or oozing, visible vessels, or blood clot adhered to the ulcer base) were identified.

\section{Study Duration}

The study duration was for three calendar years from September 2015 till august 2018.

\section{Sample Size \\ 460 patients}

\section{Inclusion Criteria}

Consecutive patients with acute peptic ulcer bleeds admitted at Medicine ward, JNIMS.

\section{Exclusion Criteria}

The following patients were excluded-

1. Proton Pump inhibitors within 2 weeks of endoscopy.

2. Antibiotics within 4 weeks of endoscopy.

3. Refused consent.

\section{Helicobacter pylori Detection Test}

Rapid Urease Test (RUT dry test, Gastrocure systems, Kolkata, India) was carried out. One biopsy specimen was taken from the mid-antrum and put in the RUT kit as per the manufacturers instruction and the result was read after 1 hour and considered to be positive when red spots appeared and negative when colour change was absent.

\section{Statistical Analysis}

Baseline characteristics were presented as mean \pm standard deviation (SD) for continuous variables and as a frequency (Percentage) for all variables. Chi square test was used where applicable.

\section{RESULTS}

During the study period, a total of 1245 patients underwent Upper GI endoscopy for Upper GI bleeds of which 514 patients were due to peptic ulcer bleeds. Of these 34 patients had recent history of taking antibiotics and 20 patients had history of taking proton pump inhibitor within four weeks of undergoing endoscopy and were excluded and so a total of 460 patients were available for analysis.

\section{Patient Characteristics}

The demographic details and disease characteristics of patients are shown in Table No.1. The mean age of patients recruited in the study was $53.4+16.8$. Males comprised of $74.13 \%$ (341). Duodenal ulcer bleeding comprised of $85 \%$ (391) of the total peptic ulcer bleeds and Gastric ulcer constituted $10.44 \%(48)$ and combined gastric and duodenal ulcer in $4.56 \%(21)$. $18.69 \%$ (86) of patients had taken NSAIDS before admission. Pre-endoscopy high dose proton pump inhibitors were prescribed in $93.91 \%$ (432) of patients.

\begin{tabular}{|c|c|}
\hline \multicolumn{2}{|c|}{ Demographics and Clinical Characteristics n (\%) } \\
\hline Age (Mean \pm SD) & $53.4 \pm 16.8$ \\
\hline Male & $341(74.13)$ \\
\hline Females & $119(25.87)$ \\
\hline Presenting Symptoms & $112(24.34)$ \\
Hematemesis & $101(21.95)$ \\
Melena & $242(52.60)$ \\
Hematemesis with Melena & $5(1.08)$ \\
\hline Haematochezia & $86(18.69)$ \\
\hline NSAID Use before Admission & $432(93.91)$ \\
\hline Pre-Endoscopic High Dose PPI Use \\
\hline \multicolumn{2}{|c|}{ Table 1. Demographics and Clinical Characteristics } \\
\hline
\end{tabular}

\section{Endoscopy Setting and Findings}

Upper GI endoscopy was performed within 12 hours in $67.82 \%$ (312), within $12-24$ hours in $7.39 \%$ (34) and 24 hours after admission in $24.79 \%$ (114). The high risk stigmata of ulcer in this study included spurting/oozing in $26.08 \%$ (120), visible vessel $29.56 \%$ (136) and overlying clot $30.62 \%$ (141).

\section{Helicobacter pylori Detection Test}

RUT was performed in all patients to diagnose $\mathrm{H}$. pylori infection. RUT was positive in 419 patients (91.08\%). Of the 48 gastric ulcer patients $82.6 \%$ (38) had positive RUT and of the 412 duodenal ulcer patients $92.47 \%$ (381) had positive RUT. The prevalence of $\mathrm{H}$. Pylori infection was higher in duodenal ulcer patients as compared to gastric ulcer patients which was found to be statistically significant $(\mathrm{P}=0.045)$.

\begin{tabular}{|c|c|}
\hline \multicolumn{2}{|c|}{ Endoscopy Setting and Findings n (\%) } \\
\hline Timing & $312(67.82)$ \\
$<12$ Hours & $34(7.39)$ \\
$12-24$ Hours & $114(24.79)$ \\
$>24$ Hours & $391(85)$ \\
\hline Location of Ulcer & $48(10.44)$ \\
Duodenal & $21(4.56)$ \\
Gastric & $8(1.73)$ \\
Gastric and Duodenal & $112(24.34)$ \\
Endoscopic Stigmata & $136(29.56)$ \\
Spurting & $141(30.65)$ \\
oozing & $23(5)$ \\
Visible Vessel & $40(8.69)$ \\
Adherent Clot & \\
Flat Pigmented Spot & Clean Based \\
\hline \multicolumn{2}{|c|}{ Table 2. Endoscopy Setting and Findings (n=460) } \\
\hline
\end{tabular}




\section{DISCUSSION}

Detecting H. pylori infection and eradicating the organism in infected patients with peptic ulcer bleeds are important to avoid recurrent bleeding. The recurrence rate reported is only $3 \%$ of the patients who received eradication treatment but as high as $20 \%$ in those treated with only antisecretory non-eradicating therapy (Without subsequent long-term maintenance antisecretory therapy. ${ }^{6,7}$

The overall prevalence rate of $\mathrm{H}$. pylori infection in this study was $91.08 \%$. When comparing the prevalence of $\mathrm{H}$. pylori infection in gastric and duodenal ulcer patients, it was found that the prevalence of $\mathrm{H}$. pylori was higher in duodenal ulcer patients as compared to gastric ulcer patients which was found to be statistically significant $(\mathrm{P}=0.045)$.

Prevalence of $\mathrm{H}$. pylori infection is higher in developing countries or areas of low socio-economic status. This study showed high prevalence $(91.08 \%)$ of $\mathrm{H}$. pylori infection in acute peptic ulcer bleeding patients. Though we do not have any data on the prevalence of $\mathrm{H}$. pylori infection in the general population, but being in a low socio economic status region we expect it to be a highly prevalent zone for $\mathrm{H}$. pylori infection.

The findings of this study is similar to another study from Vietnam conducted in 171 peptic ulcer bleeding patients where they found a H. pylori infection rate of $94.2 \% .^{8}$ But another study from Africa found a prevalence rate of 16.7 in bleeding peptic ulcer patients which was found to be much lower as compared to our study. ${ }^{9}$

One major drawback of this study is the definition of $\mathrm{H}$. pylori positivity based on a single diagnostic test. Incorporation of other diagnostic test like histology, culture and faecal antigen test would have made the study more robust.

\section{CONCLUSION}

Our study showed high $\mathrm{H}$. pylori infection rates in patients with acute peptic ulcer bleeds. The infection rate was higher in duodenal ulcer bleeds as compared to gastric ulcer bleeds which was found to be statistically significant. This exploratory study showed the importance of actively looking for $H$. pylori infection in patients with acute peptic ulcer bleeds who are likely to rebleed if not treated with $\mathrm{H}$. pylori eradication regimes.

\section{ACKNOWLEDGEMENT}

This study was done as a combined team work from all the staff working in the endoscopy room.

\section{REFERENCES}

[1] Gisbert JP, Calvet X, Feu F, et al. Eradication of Helicobacter pylori for the prevention of peptic ulcer rebleeding. Helicobacter 2007;12(4):279-86.

[2] Gisbert JP, Calvet X, Cosme A, et al. Long-term followup of 1,000 patients cured of Helicobacter pylori infection following an episode of peptic ulcer bleeding. The American Journal of Gastroenterology 2012;107(8):1197-204.

[3] Malfertheiner P, Megraud F, O'Morain CA, et al. Management of Helicobacter pylori infection - the Maastricht IV/Florence Consensus Report. Gut 2012;61(5):646-64.

[4] Dhakal OP, Dhakal M. Prevalence of Helicobacter pylori infection and pattern of gastrointestinal involvement in patients undergoing upper gastrointestinal endoscopy in Sikkim. Indian J Med Res 2018;147(5):517-20.

[5] Sharma PK, Suri TM, Venigalla PM, et al. Atrophic gastritis with high prevalence of Helicobacter Pylori is a predominant feature in patients with dyspepsia in a high altitude area. Trop Gastroenterol 2014;35(4):246-51.

[6] Gisbert J, Khorrami S, Carballo F, et al. H. pylori eradication therapy vs. antisecretory non-eradication therapy (with or without long-term maintenance antisecretory therapy) for the prevention of recurrent bleeding from peptic ulcer. The Cochrane Database of Systematic Reviews 2004;(2):CD004062.

[7] Gisbert JP, Khorrami S, Carballo F, et al. Meta-analysis: Helicobacter pylori eradication therapy vs. antisecretory non-eradication therapy for the prevention of recurrent bleeding from peptic ulcer. Alimentary Pharmacology and Therapeutics 2004;19(6):617-29.

[8] Quach DT, Luu MN, Hiyama T, et al. Early diagnosis of Helicobacter Pylori infection in Vietnamese patients with acute peptic ulcer bleeding: a prospective study. Article ID 3845067, Gastroenterology Research and Practice 2017;(2017): p. 7.

[9] Kgomo MK, Mashoesoe KS, Rheeder P. The Prevalence of Helicobacter pylori infection in bleeding and non bleeding gastric ulcers: a cross sectional case control study. Journal of Bio-analysis and Biomedicine 2016;8(4):58-62. 